\title{
Sequential magnetic mapping of bacteria loaded with Pd-Fe nanoparticles
}

\author{
James Claxton \\ Department of Physics \\ University of Oslo \\ Oslo, Norway \\ j.b.claxton@fys.uio.no \\ Dirk Linke \\ Department of Biosciences \\ University of Oslo \\ Oslo, Norway \\ dirk.linke@ibv.uio.no
}

\author{
Nadeem Joudeh \\ Department of Biosciences \\ University of Oslo \\ Oslo, Norway \\ nadeem.joudeh@ibv.uio.no \\ Pavlo Mikheenko \\ Department of Physics \\ University of Oslo \\ Oslo, Norway \\ pavlo.mikheenko@fys.uio.no
}

\author{
Anja Røyne \\ Department of Physics \\ University of Oslo \\ Oslo, Norway \\ anja.royne@fys.uio.no
}

\begin{abstract}
Magnetic nanoparticles are of widespread use in nanotechnology. One of the most unusual are magnetic palladium nanoparticles that combine magnetism with high catalytic activity. These nanoparticles could be obtained biologically by exposing bacteria to a palladium salt. Due to their small size and weak magnetism, however, it is challenging to measure their magnetic properties. One of the solutions to enhance their magnetism is to incorporate a small amount of iron atoms into them. After this procedure, the nanoparticles together with bacteria can be embedded in resin and characterized by the technique of magnetic force microscopy. This technique allows imaging cross-sections of the bacteria with nanoparticles, but cannot give information from the depth of the sample. Here we report on an approach partially solving this problem. Its novelty lies in measurements of consecutive thin slices of resin, which allows mapping cross-sections of individual bacteria and different parts of the material surrounding the same bacterium. An interesting observed feature is the formation of magnetic chains of nanoparticles outside of the bacteria.
\end{abstract}

Keywords - Nanoparticles, magnetic mapping, palladium, iron, bacteria, magnetic force microscopy

\section{INTRODUCTION}

Magnetic nanoparticles are becoming indispensable tools in nanotechnology. Their applications range from magnetic recording media [1] to delivery of drugs [2] and treatment of cancer [3]. Special attention is attracted to palladium (Pd) nanoparticles that become magnetic when their size is in the range of a few nanometers [4,5]. Combined with excellent catalytic properties, these nanoparticles are especially efficient in cancer treatment [6]. A biological method for producing $\mathrm{Pd}$ nanoparticles in very large amounts is to introduce bacteria to a Pd salt solution [7]. In their exchange with the environment, bacteria can supply electrons for redox reactions, and efficiently reduce $\mathrm{Pd}$ salts from solution due to very high redox potential of the latter [8,9]. Adding iron $(\mathrm{Fe})$ salt to the solution allows obtaining $\mathrm{Pd}$ particles that incorporate a small amount of $\mathrm{Fe}$, which strongly increases their magnetism [10]. It could be straightforward to use these $\mathrm{Pd}-\mathrm{Fe}$ nanoparticles for applications, but it is not easy to measure their magnetic properties. Here a technique is reported allowing doing this with the help of magnetic force microscopy (MFM).

\section{EXPERIMENTAL}

\section{A. Magnetic force microscopy}

Magnetic force microscopy (MFM) [11-13] is a technique allowing mapping magnetic properties of a sample with a magnetic tip, which is scanned above its surface. In order to distinguish between magnetic and Van der Waals forces, which are acting on the tip at small distances, a twopass scanning technique is used [11,12]. In the first pass, topography close to the surface is mapped. In the next scan, the probe is moved along a path following the measured topography, but at a larger height, so that the probe-sample distance is kept constant. If the height is large enough, Van der Waals forces become weak, and the pure magnetic response can be measured. According to [11,13,14], shift in the phase of oscillations, if AC mode is used, is proportional to the gradient of force acting on the tip. The measurements were done using JPK NanoWizard 4.0 in AC mode at a frequency of about $74 \mathrm{kHz}$ in an applied field of $0.58 \mathrm{~T}$, created by a permanent magnet within the sample holder. The probes used were manufactured by Nanosensors, model type PPP-MFMR-10, with a tip radius of approximately 50 nm.

\section{B. Production and preparation of samples}

A single colony of Escherichia coli BW25113 strain was inoculated into $10 \mathrm{ml}$ lysogeny broth (LB) medium in a test tube overnight at $37{ }^{\circ} \mathrm{C}$ while shaking it at $200 \mathrm{rpm}$. On the next day, $1 \mathrm{ml}$ of this medium was used to inoculate $49 \mathrm{ml}$ fresh LB medium in a $250 \mathrm{ml}$ flask. The flask was also incubated at $37{ }^{\circ} \mathrm{C}$ while shaking at the same speed until the optical density (O.D.600) reached 0.5. The medium was transferred to a $50 \mathrm{ml}$ falcon tube and centrifuged at $4250 \mathrm{~g}$ for 10 mins. The supernatant was removed, and the pellet was resuspended in $10 \mathrm{ml} 20 \mathrm{mM} \quad \mathrm{pH} \quad 7 \quad 3-(\mathrm{N}-$ morpholino)propanesulfonic acid (MOPS) buffer. This washing step was done two more times, except for the last round the pellet was resuspended in $8 \mathrm{ml} 20 \mathrm{mM} \mathrm{pH} 7$ MOPS buffer. $1 \mathrm{ml}$ of this suspension was transferred to a 1.5-ml Eppendorf tube. $1 \mathrm{mM}$ of sodium tetrachloropalladate $\left(\mathrm{Na}_{2} \mathrm{Cl}_{4} \mathrm{Pd}\right)$ and $1 \mathrm{mM}$ of iron III chloride $\left(\mathrm{FeCl}_{3}\right)$ (both dissolved in $0.01 \mathrm{M}$ nitric acid) were added to the tube, shaken well by hand and incubated for 1 hour. Then, $10 \mathrm{mM}$ 
sodium formate was added to the tube to start the reduction. The suspensions turned black within 20 minutes.

Pd-Fe loaded bacteria were washed twice with distilled water by centrifuging at $5900 \mathrm{~g}$ for $2 \mathrm{~min}$. After the second centrifugation step, the pellet was resuspended in $0.5 \mathrm{ml}$ fixative solution (4\% paraformaldehyde and $2 \%$ glutaraldehyde (w/v) dissolved in $20 \mathrm{mM} \mathrm{pH} 7$ MOPS buffer) and left for 10 minutes at room temperature. Another round of fixation was done with the same concentration of the solution, and the sample was left in the fridge overnight. On the next day, the sample was washed twice with distilled water by centrifuging it at $3500 \mathrm{~g}$ for 10 minutes. Then, the sample was dehydrated by using an ascending ethanol concentration series $(50,70,90,96$ and 100\%). The sample was incubated with each concentration for 10 min except the last concentration. For the last concentration, four rounds of dehydration were done, each taking $15 \mathrm{~min}$. Next, one additional round with $100 \%$ acetone was applied for 15 minutes. Lastly, one round of 1:1 acetone to epoxy was done overnight leaving the cap off, therefore, the epoxy concentration slowly increased. The next day, the sample was centrifuged at $3500 \mathrm{~g}$ for 10 minutes, then, $0.5 \mathrm{~mL}$ of $100 \%$ epoxy was added and incubated for 1 hour at room temperature. Afterwards, the tube was moved to the oven and incubated at $60{ }^{\circ} \mathrm{C}$ for 3 days. The embedded sample was then sliced with a diamond knife into 0.5-1 $\mu \mathrm{m}$ thin slices. The slices were moved in a water droplet, put on smooth glass slides and left for the water to evaporate. After evaporation of water, the slices firmly stick on the slides, ready for MFM measurements. The thickness of the slices was kept above $500 \mathrm{~nm}$. The reason for this was that the sliced resin would bend and fold if slices are too thin. Very flat surfaces were found to be of key importance for MFM imaging at high resolution.

\section{Sequential MFM Imaging}

With accurate cutting of the slices and setting them to the glass, they form a chain, in which each next slice was below (or above) the previous one in the sliced volume of resin. A fragment of a chain of slices is shown in Fig. 1. Imaging slices individually allows us to characterize different crosssections of the resin, which may include cuts of the same bacteria and give information about the three-dimensional distribution of nanoparticles around them. Collecting different parameters of tip oscillation during the scans allows reconstructing both topography and magnetic properties of bacteria and nanoparticles.

Finding the same group of bacteria on each slice requires considerable effort. Its approximate position was determined using an optical microscope with a camera above the MFM

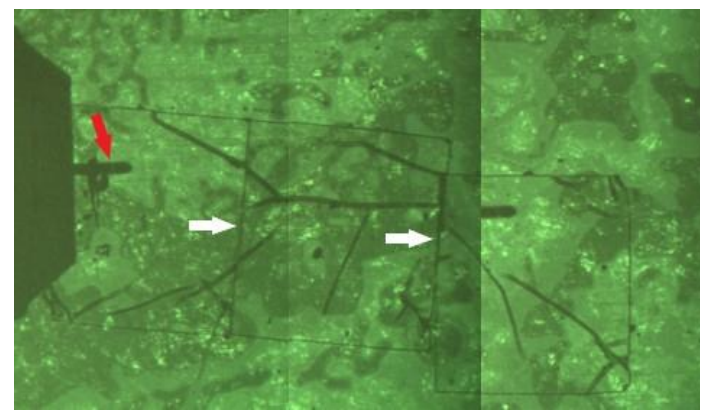

Fig. 1. Optical image of the slices of bacteria prepared for MFM imaging. The red arrow indicates the probe cantilever and the white arrows indicate the edges between slices of resin. stage designed to see cantilever above a slice. When scanning the surface, large areas $\left(90 \times 90 \mu^{2}\right)$ were initially mapped in order to compare features on a large scale. Scanning an area larger than $20 \times 20 \mu \mathrm{m}^{2}$ at typical rate of scan of $1 \mathrm{~Hz}$ is usually not recommended, as the tip moves across the surface too rapidly. Therefore, to achieve desirable resolution, the scan rate was reduced to $0.2 \mathrm{~Hz}$ doing the measurement much slower than usually.

\section{RESULTS AND DISCUSSION}

\section{A. Magnetic mapping of Pd-Fe nanoparticles}

Magnetic mapping was done with the two-pass technique at an optimal distance of $20 \mathrm{~nm}$ above the surface, at which Van der Waals forces become weak, but magnetic forces are still strong. Fig. 2 shows a selected magnetic map with clearly visible Pd-Fe nanoparticles concentrated around the cross-section of bacteria and forming extended magnetic chains and clusters. The nanoparticles are also seen on the outline of the bacteria. In the figure, the phase shift is proportional to the gradient of the magnetic force acting on the tip. Negative (dark) contrast represents attraction of the tip to a nanoparticle. In a few places, attraction is replaced by repulsion, when the tip is coming too close to the surface and the Van der Waals forces become a dominant force.

\section{B. Sequential MFM Imaging}

The sequential imaging was done on the slices of resin shown in Fig. 1. Fig. 3 gives amplitude maps (measured during the first pass) of a $90 \times 90 \mu \mathrm{m}^{2}$ area selected in the same place on each of the three slices. The cantilever in Fig. 1 shows an approximate position inside the scanned area in the left-hand slice. In Figs. 3a, 3b and 3c, the same features can be seen confirming that the imaged clusters of bacteria belong to the same place, and even that three cross-sections of the same bacteria could be found. The images differ slightly since some bacteria begin or end in the imaged slices. Specifically, the bacteria embedded with their long axis parallel to the surface are less likely to be found in the sequential slices. By imaging smaller regions, individual bacteria can be found in each slice. Fig. 4 shows higher resolution $20 \times 20 \mu \mathrm{m}^{2}$ maps of the amplitude of oscillations in each slice of the resin. In these maps, a region of interest was a bacterium (outlined by dashed lines) with the chains of nanoparticles attached to it. These scans with even higher magnification are shown in Fig. 5. In Fig. 5a and 5b, the cross-sections of the bacterium are visible at the top with the attached nanoparticles. In Fig. 5c, the bulging at the edge of

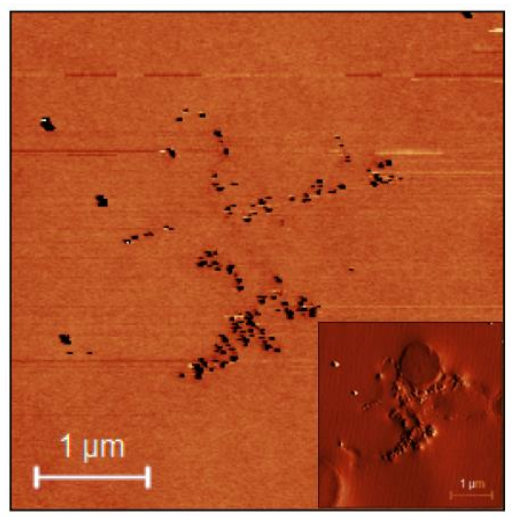

Fig. 2. Magnetic map of a cross-section of bacteria with chains of magnetic nanoparticles. The magnetic map displays the phase shift at lift height of 20 $\mathrm{nm}$. Inset: Amplitude map representing topography of the same area. 
the bacterium is visible. These maps suggest that imaging bacteria and nanoparticles is possible in a three-dimensional way, allowing detailed topographical characterization of them. Fig. 6 shows the same areas as Fig. 5, but displays the shift in the phase of oscillations measured during the second pass. Negative (dark) and positive (light) contrast indicate attractive and repulsive interaction, respectively, between the sample and the tip. Most nanoparticle-tip interactions appear

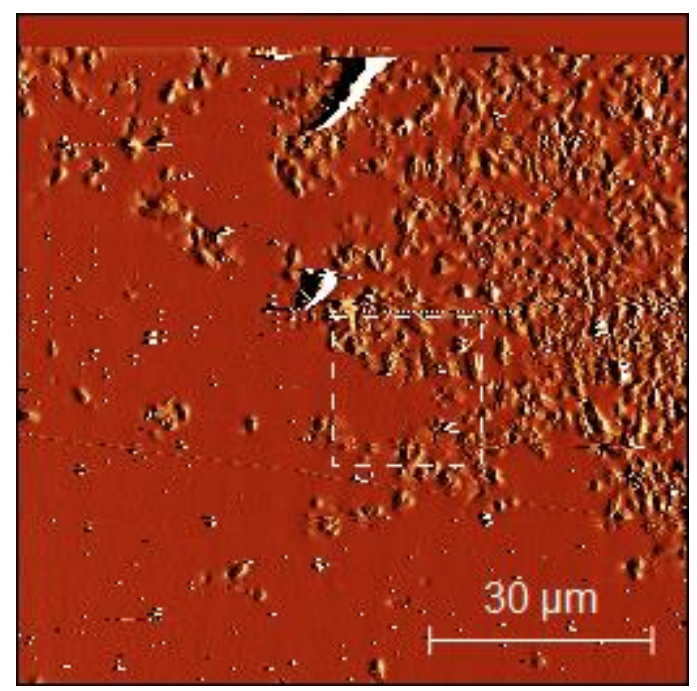

a)

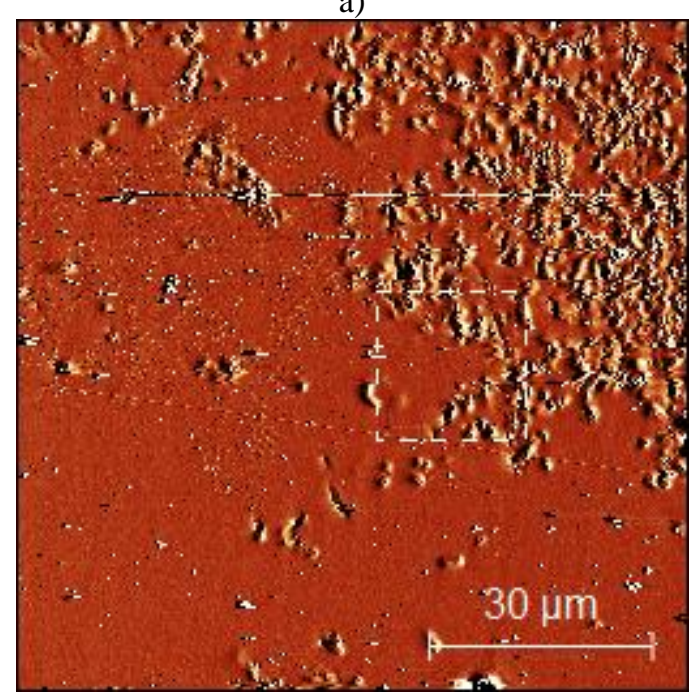

b)



c)

Fig. 3. Amplitude maps of three consecutive slices of resin measured during the first pass. An area of $90 \times 90 \mu^{2}$ has been imaged.

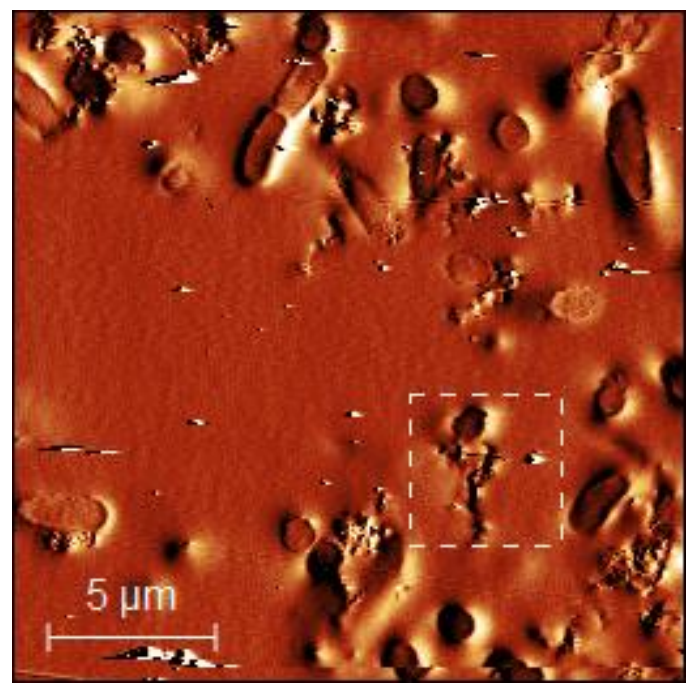

a)

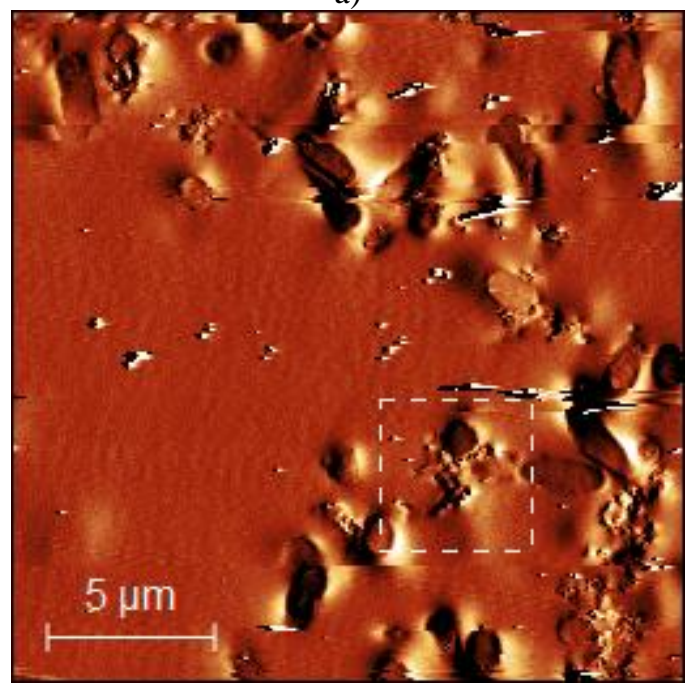

b)



c)

Fig. 4. Amplitude maps of three slices of resin measured during the first pass. A $20 \times 20 \mu \mathrm{m}^{2}$ area has been imaged to compare individual bacteria. The location of these scans is indicated by the squares surrounded by dashed lines in Fig. 3. 


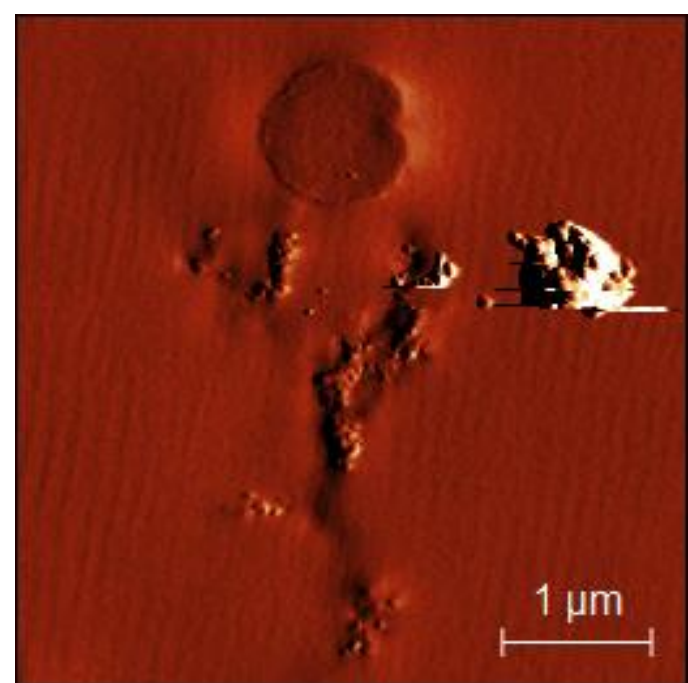

a)



b)



c)

Fig. 5. Amplitude maps of three slices of resin measured during the first pass. Nanoparticle chains are visible connected to bacteria. Location of scans is indicated by the squares surrounded by dashed lines in Fig. 4.

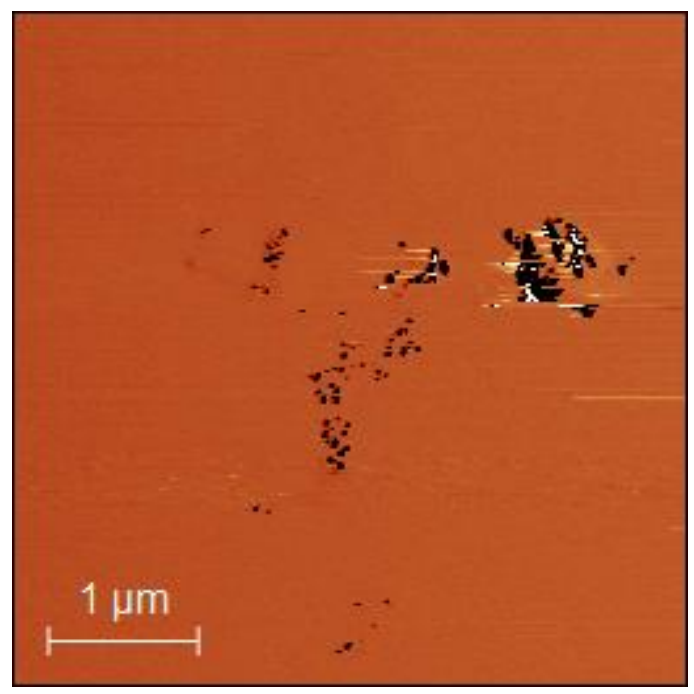

a)

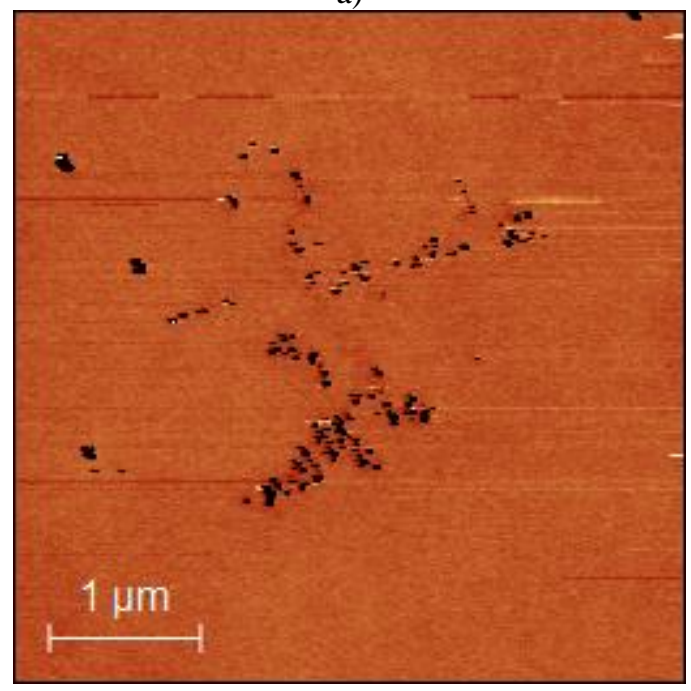

b)

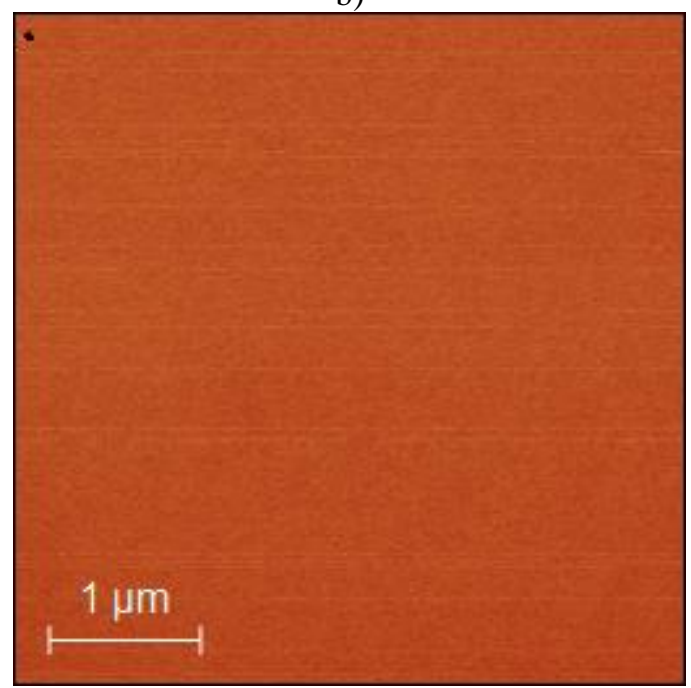

c)

Fig. 6. Phase maps of three slices of resin, measured during the second pass at lift height of $20 \mathrm{~nm}$, in the same location as Fig. 5. 
in negative contrast, indicating that the probe and nanoparticles are attracted to each other. On the other hand, there are clusters of nanoparticles (Fig. 6a), which show bright positive contrast, suggesting strong repulsion. The aim of this figure is to present the pure magnetic signal coming from the magnetic interaction between the sample and the tip. The clusters and chains of nanoparticles, the formation of which is currently not well understood, are found outside of the bacteria. During the sample preparation, the solution was washed several times. However, the chains and clusters remain attached to the bacteria, suggesting a strong chemical or magnetic interaction between the nanoparticles themselves and the bacteria and nanoparticles.

Images in Fig. 6 were recorded at lift height set to 20 $\mathrm{nm}$. This height was selected experimentally for the following two reasons. Firstly, the bacterial cell walls, which are visible in Fig. 5, are not visible in Fig. 6, suggesting that atomic forces from mainly non-magnetic material (bacteria still tend to accumulate weakly-magnetic pure Pd particles between the outer and inner membranes) are not acting on the tip. Secondly, it was found that mounting a non-magnetic probe and repeating the same scans at $20 \mathrm{~nm}$ above the sample resulted in negligible interaction between the sample and the tip. This suggests that $20 \mathrm{~nm}$ from the sample surface to the tip apex is a sufficient enough distance to measure a pure magnetic response with negligible atomic and electrostatic interaction. Fig. 7 shows a three-dimensional representation of the cross-sections. The approximate position of the bacteria is indicated by the blue ellipsoids.

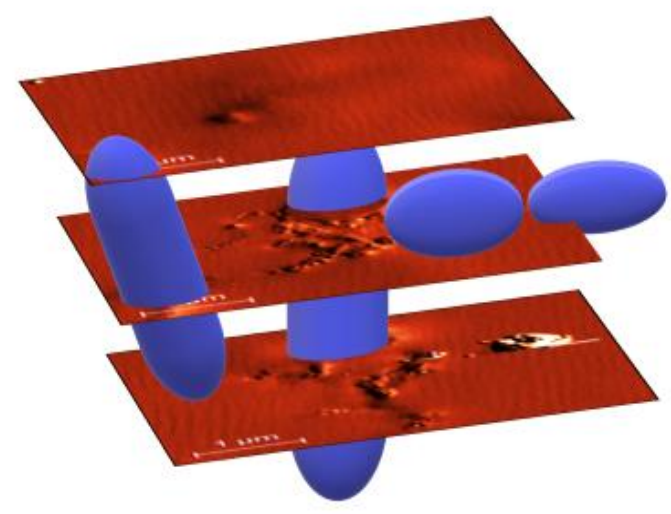

Fig. 7. A 3D representation of the slices of bacteria shown in Fig. 5. The sequence of slices is changed to better show bulging in the top layer.

\section{CONCLUSIONS}

In conclusion, by embedding bacteria and biologically produced palladium-iron nanoparticles into resin, slicing it into several cross-sections and imaging the sequence of slices by magnetic force microscopy, a three-dimensional view of the structures was reconstructed. This method partially solves the problem of characterization restricted to the surface of the material only. In this case, the method has enabled nanoparticles and bacterial cross-sections deeper in the resin to be mapped. Unusual nanoparticle chains and clusters were observed extending from the bacterial crosssections. The optimal height for mapping magnetic response was found, which isolates the tip-sample magnetic interaction from the atomic interactions. This allows the magnetic characterization of nanoparticles to be performed without being affected by the short-range atomic forces. The iron atoms were added to enhance the magnetic response of the palladium nanoparticles, which now could be used in various applications with additional functionality allowing to recover them magnetically after the use.

\section{ACKNOWLEDGMENT}

This work is part of the Project "BEDPAN - Bioengineered Palladium Nanoparticles", funded by the Research Council of Norway, RCN294605.

\section{REFERENCES}

[1] J. Wang, "FePt magnetic nanoparticles and their assembly for future magnetic media," Proceedings of the IEEE, vol. 96, pp. 1847-1863, December 2008

[2] S. Mirza, M. S. Ahmad, M. I. A. Shah, and M. Ateeq, "Magnetic nanoparticles: drug delivery and bioimaging applications," in Metal Nanoparticles for Drug Delivery and Diagnostic Applications. Amsterdam: Elsevier, 2020, pp. 189- 213.

[3] J. Jose, R. Kumar, S. Harilal, G. E. Mathew, D. G. T Parambi ,A. Prabhu, M. S. Uddin, L. Aleya, H. Kim, B. Mathew, "Magnetic nanoparticles for hyperthermia in cancer treatment: an emerging tool," Environmental Science and Pollution Research, vol. 27, pp. 19214-19225, December 2019.

[4] T. Shinohara, T. Sato, and T. Taniyama, "Surface ferromagnetism of Pd fine particles," Physical Review Letters, vol. 91, 197201, November 2003.

[5] S. Angappane, J. Park, Y. Jang, T. Hyeon, and J.-G. Park, "Magnetic Pd nanoparticles: effects of surface atoms," Journal of Physics: Condensed Matter, vol. 20, 295209, July 2008.

[6] M. A. Miller, B. Askevold, H. Mikula, R. H. Kohler, D. Pirovich, and R. Weissleder, "Nano-palladium is a cellular catalyst for in vivo chemistry," Nature communications, vol. 8, pp. 1-13, July 2017.

[7] S. Tarver, D. Gray, K. Loponov, D. B. Das, T. Sun, and M. Sotenko, "Biomineralization of $\mathrm{Pd}$ nanoparticles using phanerochaete chrysosporium as a sustainable approach to turn platinum group metals (pgms) wastes into catalysts," International Biodeterioration and Biodegradation, vol. 143, 104724, September 2019.

[8] S. Jang, S. A. Hira, D. Annas, S. Song, M. Yusuf, J. C. Park, S. Park, and K. H. Park, "Recent novel hybrid $\mathrm{Pd}-\mathrm{Fe}_{3} \mathrm{O}_{4}$ nanoparticles as catalysts for various C-C coupling reactions," Processes, vol. 7, 422, July 2019.

[9] K. Deplanche, I. Caldelari, I. P. Mikheenko, F. Sargent and L. E. Macaskie, "Involvement of hydrogenases in the formation of highly catalytic $\mathrm{Pd}(0)$ nanoparticles by bioreduction of $\mathrm{Pd}(\mathrm{II})$ using Escherichia coli mutant strains," Microbiology, vol. 156, pp. 26302640, June 2010.

[10] L. E. Macaskie, V. S. Baxter-Plant, N. J. Creamer, A. C. Humphries, I. P. Mikheenko, P. M. Mikheenko, D. M. Penfold and P. Yong, "Applications of bacterial hydrogenases in waste decontamination, manufacture of novel bionanocatalysts and in sustainable energy," Biochem. Soc. Trans., vol. 33, pp. 76-79, February 2005.

[11] D. Passeri, C. Dong, M. Reggente, L. Angeloni, M. Barteri, F. A. Scaramuzzo, F. De Angelis, F. Marinelli, F. Antonelli, F. Rinaldi, C. Marianecci, M. Carafa, A. Sorbo, D. Sordi, I. WCE Arends, and M. Rossi, "Magnetic force microscopy. Quantitative issues in biomaterials," Biomatter, vol. 4, e29507, July 2014.

[12] G. Cordova, B.Y. Lee, and Z. Leonenko, "Magnetic force microscopy for nanoparticle characterization," NanoWorld Journal, vol 2, pp. 1014, April 2016.

[13] B. Torre, G. Bertoni, D. Fragouli, A. Falqui, M. Salerno, A. Diaspro, R. Cingolani, and A. Athanassiou, "Magnetic, force microscopy and energy loss imaging of superparamagnetic iron oxide nanoparticles," Sci. Rep., vol.1, 202, December 2011.

[14] Sarid D, Scanning Force Microscopy. New York, USA: Oxford University Press, 1994. 